\title{
A Procedure for Development and Execution of Process-Based Composite Web Services
}

\author{
Dimka Karastoyanova and Alejandro Buchmann \\ Technische Universität Darmstadt \\ Department of Computer Science \\ Hochschulstrasse 10, 64289 \\ Darmstadt, Germany \\ dimkalgkec.tu-darmstadt.de \\ buchmannainformatik.tu-darmstadt.de
}

\begin{abstract}
The paper proposes a methodology for development and execution of Web service compositions. It uses a common process meta-model and accommodates approaches for code and functionality reuse applying templates, and dynamic selection and invocation of participating WSs at run time.
\end{abstract}

\section{Introduction}

Web service (WS) technology targets the integration of applications across organizational boundaries and over the Web. It is not yet a mature middleware technology. There is no standard methodology to guide the creation and execution of WS-based compositions, called WS-flows, and to accommodate approaches addressing process development automation, flexibility and adaptability. In this paper we introduce the main steps of such a methodology, based on WS-flows life cycle [3].

\section{Procedure for Development and Execution of WS-flows}

To meet the requirements on the design of WS-flows we propose a simple procedure, presented in Fig. 1. Each phase prescribes an approach addressing different aspects of a process definition [3]. During the process template modeling and assembly phase templates are modeled using the constructs of a common meta-model and are assembled with additional business logic to produce abstract process definitions. Templates are units of code and functionality reuse. The resulting process definitions avoid specifying any references to WS instances, and in a more complex approach avoid references to WSs portTypes. Process definition generation phase is used to transform the templates created in the previous phase into executable process definitions using meta-programming applications like code generators, or XML transformations. Real flexibility of the WS-flows can be achieved if the commitment to a specific process definition language is deferred to the latest possible transformation. 


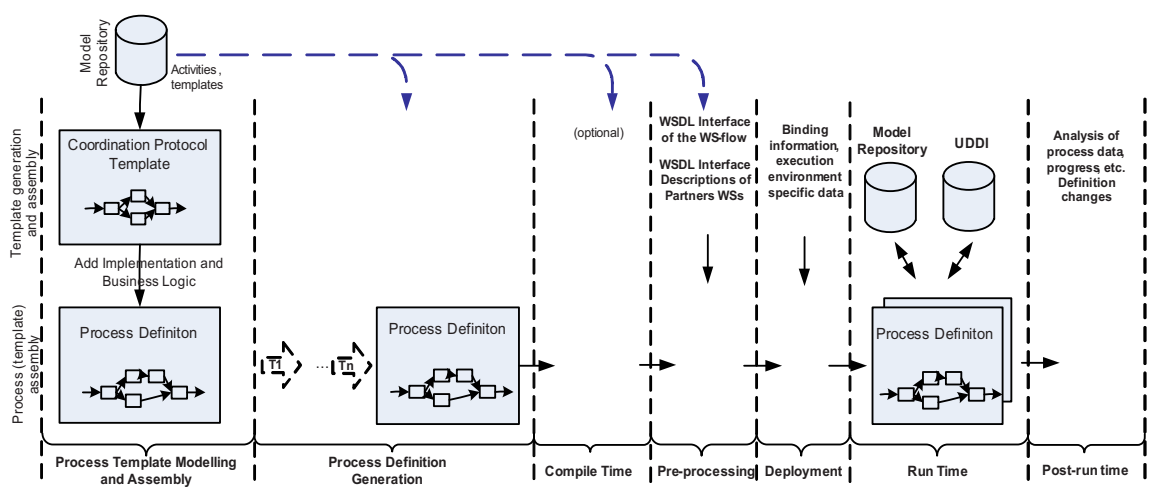

Fig. 1. Procedure for development and execution of WS-flows.

The compile time and pre-processing time are optional phases but in some cases they might be necessary depending on the targeted definition language. Upon deployment the WS-flow definition is enriched with execution environment specific data, and with details about the participating WSs (e.g. BPEL4WS [2]). During execution time process instances are created and executed. The use of a common process model enables dynamic selection of WSs, based on their QoS characteristics, and WS invocation at run time. A more flexible solution advocates the use of reflective activities to allow changes to be made in the process definition at run time (Fig. 1). The information gathered during run time can later be used in the post-run time phase to analyze the process progress and logic and change it accordingly.

\section{Conclusion}

We introduce a procedure for development and execution of WS-flows. It is based on the use of a common process model. During the build time phases a WS-flow definition is modeled and generated from templates. The procedure relies strongly on enabling process definition reuse and technology leverage; it reduces manual work and shortens development time. Automating the procedure is instrumental for its success and depends on the existence of appropriate tools for template modeling and transformation. The methodology can be used to create process definitions in multiple languages, e.g., BPEL [2] and BPML [1]. It facilitates the development of flexible WS-flows by providing means to allow dynamic selection and invocation of WSs during process execution.

\section{References}

1. Arkin, A. et al., "Business Process Modeling Language”, BPMI.org, 2002.

2. Curbera, F. et al., "Business Process Execution Language for Web Services 1.0”, 2002.

3. Karastoyanova, D., A Methodology for Development of Web Service-based Business Processes, In Proceedings of AWESOS 2004, April 2004. 